drawing conclusions about the evolutionary history of shared features.

Caroline B. Albertin and Clifton W. Ragsdale are in the Department of Neurobiology,

The University of Chicago, Chicago,

Illinois 60637, USA.

e-mails:calbertin@uchicago.edu; cragsdale@uchicago.edu

1. Cornell, R. A. \& Von Ohlen, T. Curr. Opin. Neurobiol. 10, 63-71 (2000).

2. Denes, A. S. et al. Cell 129, 277-288 (2007)

3. Martín-Durán, J. M. et al. Nature 553, 45-50 (2018).

4. Dohrn, A. \& Ghiselin, M. T. Hist. Phil. Life Sci. 16, 3-96 (1994).

5. McGinnis, W. \& Krumlauf, R. Cell 68, 283-302 (1992).
6. De Robertis, E. M. \& Sasai, Y. Nature 380, 37-40 (1996).

7. Cannon, J. T. et al. Nature 530, 89-93 (2016)

8. Lowe, C. J. et al. PLoS Biol. 4, e291 (2006).

9. Scimone, M. L., Kravarik, K. M., Lapan, S. W. \& Reddien, P. W. Stem Cell Rep. 3, 339-352 (2014).

10.Striedter, G. F. et al. Brain Behav. Evol. 83, 1-8 (2014).

This article was published online on 13 December 2017.

\section{Ultrasound approach tracks gut microbes}

\section{Monitoring microbes that live deep inside the gut is a challenge. Engineering bacteria to express structures that can be tracked by ultrasound offers a way to locate such cells in vivo, and might have clinical implications. SEE LETTER P.86}

\section{RICARD SOLÉ \& NURIA CONDE-PUEYO}

A microbial ecosystem exists inside you that is as rich and complex as the rainforest. Like the rainforest, this ecosystem contains inaccessible realms that are usually hidden from view. When trying to observe the living gut, a major problem is that light-based imaging techniques can monitor only a limited depth below the surface. However, on page 86 , Bourdeau et al. ${ }^{1}$ report an ultrasound approach for exploring this inner world that they use to map the in vivo location of specific microbial-cell populations. Some medical approaches currently in use or being developed introduce bacterial cells as a therapy for gut disease or cancer, so this ultrasound technique might be adapted for clinical use to determine whether such cells have reached the desired location.

Microbial communities have been coevolving with humans over millions of years ${ }^{2}$, and they display notable spatial and temporal regularities in their organization. This natural ecosystem assembles at birth, develops, responds to perturbations and stress, and can sometimes collapse. Yet determining the laws and fragilities of life deep within the gut has been difficult, and even some of the best wholebody imaging techniques available can reveal structures at depths of only centimetres below the surface ${ }^{3,4}$.

Bourdeau and colleagues offer an innovative solution. Ultrasound imaging has so far mainly been used to assess tissues, but the authors reveal that it can also be used to efficiently track populations of bacterial cells that have been genetically engineered to express what they term acoustic reporter genes. These encode components that form intracellular, protein-enclosed, gas-filled structures called gas vesicles, which are naturally present in many microorganisms, in which they control buoyancy in aqueous environments ${ }^{5}$.

Ultrasound detection involves directing pulses of sound waves towards a sample and monitoring the reflected echoes, which are affected by density differences in the substances that the sound passes through. Gas vesicles scatter sound waves, and organisms containing them can be monitored using ultrasound ${ }^{6}$. Pressure pulses above a certain level cause gas-vesicle collapse; therefore, ultrasound signals that disappear after such pulses can be inferred to have originated from gas vesicles ${ }^{6}$, an approach that could be used to enhance signal detection above background levels (Fig. 1).

There had been no previous tests to discover whether cells that do not normally form gas vesicles could be genetically engineered to do so, allowing such cells to be monitored by microorganism currently being used or developed as therapeutics to express gas-vesicle components. One of these microbes was a nonpathogenic strain of the bacterium Escherichia coli that is given to some people who have a gut infection ${ }^{7}$. Another was Salmonella enterica Typhimurium bacteria, which can invade ultrasound. Bourdeau et al. engineered types of


Figure 1 | Using ultrasound to monitor the in vivo dynamics of cell populations in space and time. a, Bourdeau et al. ${ }^{1}$ genetically engineered bacteria to express what they term acoustic response genes (ARG), which encode the components of hollow structures called gas vesicles that scatter sound waves and generate an echo that can be detected by ultrasound. Pressure-pulse application causes gasvesicle collapse and disappearance of the ultrasound signal, which can be used to improve signal detection when tracking the location of cells containing gas vesicles. This approach enables in vivo monitoring of a cell population deep within the mouse gut that cannot be tracked by light microscopy. $\mathbf{b}$, The authors engineered two types of gas vesicle (red and blue) that collapse at different pressure-pulse levels, enabling cells containing these vesicles to be distinguished using ultrasound. One possible application of this work might be to introduce two bacterial strains that each contain one type of these gas vesicles into a mouse. This would enable non-invasive in vivo temporal and spatial monitoring of the dynamics of two distinct bacterial populations in the gut in regions such as the small intestine or colon. 
tumours. Mouse models of tumour invasion by S. enterica Typhimurium are being investigated to determine the potential for using such bacteria to release tumour-killing drugs ${ }^{8,9}$.

The authors introduced engineered bacteria that expressed gas vesicles into the mouse gut and showed that the ultrasound-imaging technique works efficiently even for highly diluted cellular populations - signals were detected for $E$. coli cells present at a concentration of $5 \times 10^{7}$ cells per millilitre. The authors also demonstrated that they could engineer bacterial strains that generate distinguishable ultrasound signals, enabling two different bacterial populations to be monitored simultaneously by using strains containing gas vesicles that collapse at different pressure-pulse levels.

The authors compared their ability to monitor the location of engineered bacteria using either ultrasound or a method that tracks bacteria expressing a light-emitting molecule, using strains that live in a deep internal gut region that is difficult to visualize by optical methods. The ultrasound approach outperformed its luminescent counterpart, and ultrasound signals from engineered bacteria provided a high level of spatial resolution and reached deep areas that could not be monitored by a luminescence-based approach. Using ultrasound, the authors detected bacteria coating the surface of the colon and present at cell concentrations similar to those used in therapeutic treatments.

In vivo imaging systems that enable realtime monitoring of tumour-infiltrating luminescent bacteria can effectively monitor bacterium-associated tumours just below the skin in mice ${ }^{9,10}$, but are of little use for monitoring more-internal anatomy. Bourdeau and colleagues' ultrasound approach provides good images of engineered strains of S. enterica Typhimurium that reside deep within an internal murine tumour (an ovarian adenocarcinoma) that developed from transplanted human ovarian-cancer cells.

This ultrasound technique might also be helpful for the validation and tuning of approaches that use engineered bacterial cells to target tumours. In vivo imaging is an important part of assessing these treatments in animal models, including determining the correct dosage and estimating treatmentresponse times. Even at this proof-of-concept level, there is enormous promise that this noninvasive method might be used to monitor the effect of a bacterium-based cancer therapy in an individual over time. This work might also offer a tool for the optimization of other therapies and diagnostics being developed in which a synthetic-biology approach is used to engineer cells to have biological pathways that are not normally present in a particular cell type ${ }^{11}$.

Moreover, Bourdeau and colleagues' work might be complemented by another sound-based imaging technique, called photoacoustic imaging. In this approach, light or radio-frequency pulses trigger a thermal expansion of target tissues that generates acoustic waves ${ }^{12}$. Integrating photoacoustic imaging with the authors' method could allow the precise location of bacteria to be determined alongside detailed information of the surrounding tissue in vivo.

Other extensions and applications of the work by Bourdeau and colleagues can be envisaged. For example, engineered groups of bacteria ${ }^{13,14}$ might be designed to produce an ultrasound signal in response to specific ranges of physiological and environmental conditions in the gut. And bacterial cells engineered to respond if they interact with gut cells might help to trace the gut's functional biogeography. The ability to selectively control the expression of the acoustic response genes could be helpful in designing experiments to monitor how newly introduced bacteria colonize the gut or to observe the destruction of bacterial pathogens over space and time during therapy.

Perhaps this new technique could also be used to study systems beyond the body, such as the microbial ecosystems in healthy or damaged soil habitats. The soil can have a rich microbial community, and the spatial ecology of soil microbes is not fully understood ${ }^{15}$. Charles Darwin's image of a "tangled bank" of complex organismal interactions is relevant to both the ecological networks in the soil and the complexity of the cellular interactions in the gut. Flexible investigation tools are needed to understand these types of ecology, and future studies building on the work of Bourdeau and colleagues to report precise, acoustic-based imaging of the spatial dynamics of cells might be a crucial step forward.

Ricard Solé and Nuria Conde-Pueyo are at ICREA-Pompeu Fabra University, 08003 Barcelona, Spain. R.S. is also at the Santa Fe Institute, Santa Fe, New Mexico, USA.

e-mails: ricard.sole@upf.edu; nuria.conde@upf.edu

1. Bourdeau, R. W. et al. Nature 553, 86-90 (2018).

2. Walter, J. \& Ley, R. Annu. Rev. Microbiol. 65 , 411-429 (2011).

3. Berlec, A., Završnik, J., Butinar, M., Turk, B. \& Štrukelj, B. Microb. Cell Fact. 14, 181 (2015).

4. Hong, G., Antaris, A. L. \& Dai, H. Nature Biomed. Eng 1, 0010 (2017).

5. Walsby, A. E. Microbiol. Rev. 58, 94-144 (1994).

6. Shapiro, M. G. et al. Nature Nanotechnol. $\mathbf{9}$, 311-316 (2014).

7. Sonnenborn, U.\& Schulze, J. Microb. Ecol. Health Dis. 21, 122-158 (2009)

8. Din, M. O. et al. Nature 536,81-85 (2016).

9. Danino, T., Lo, J., Prindle, A., Hasty, J. \& Bhatia, S. N. ACS Synth. Biol. 1, 465-470 (2012)

10. Weissleder, R. Nature Rev. Cancer 2, 11-18 (2002).

11. Weber, W. \& Fussenegger, M. Nature Rev. Genet. 13, 21-35 (2012)

12.Xu, M. \& Wang, L. V. Rev. Sci. Instrum. 77, 041101 (2006).

13. Brenner, K., You, L. \& Arnold, F. H. Trends Biotechnol. 26, 483-489 (2008)

14.Macía, J., Posas, F. \& Solé, R. V. Trends Biotechnol. 30, 342-349 (2012).

15.Ettema, C. H. \& Wardle, D. A. Trends Ecol. Evol. 17, 177-183 (2002).

\section{Escape from senescence boosts tumour growth}

Some chemotherapies block cancer growth by driving tumour cells into a state of cell-division arrest termed senescence. It emerges that such cells have a boosted capacity to drive tumour growth if they exit senescence. SEE LETTER P.96

\section{JAN PAUL MEDEMA}

$\mathrm{I}$ cells encounter certain types of stress, they can enter a state of cell-division arrest termed senescence ${ }^{1}$, which is usually thought to be irreversible. Senescence protects organisms from potentially dangerous cellular proliferation, for example by preventing cell division after severe DNA damage. Many anti-cancer therapies cause cancer-cell senescence, which is considered to be a positive outcome of such treatment. However, Milanovic et al. ${ }^{2}$ reveal on page 96 the unexpected twist that chemotherapyinduced senescence might generate tumour cells that have an enhanced potential to drive tumour growth if they exit senescence.
Senescence induction has been studied intensively for decades. The phenomenon was first described in fibroblast cells grown in vitro, and entry into the senescent state in this context was considered to be a hallmark of cellular ageing ${ }^{3}$. Subsequent research has revealed that the induction of senescence is a cellular response that occurs during both physiological and pathological processes ${ }^{1}$.

The protein p53 is one of the key proteins that can act as a cellular sensor and drive a cell to enter senescence. It responds to DNA damage, and its action can cause permanent cell-cycle arrest by activating the proteins p16 ${ }^{\text {INK4a }}$ and $\mathrm{p} 21$. A senescent state can also be promoted by addition of methyl groups to specific amino-acid residues on histone 